UCRL-ID-117149

\title{
Continental Seismic Events Observed by the MPL Vertical DIFAR Array
}

\author{
D. B. Harris \\ G. D'Spain
}

November 1993

This is an informal report intended primarily for internal or limited external distribution. The opinions and conclusions stated are those of the author and may or may not be those of the Laboratory. 


\section{DISCLAIMER}

This document was prepared as an account of work sponsored by an agency of the Uniled Slates Government. Neither the United States Government nor the University of California nor any of their employees, makes any warranty, express or implied, or assumes an y legal liability or responsibility for the accuracy, completeness, or usefulness of an y information, apparalus, product, or process disclosed, or represents that its use would not infringe privalely owned rights. Reference herein to any specific commercial products, process, or service by trade name, trademark, manufacturer, or otherwise, does not necessarily constitute or imply its endorsement, recommendation, or favoring by the United States Government or the University of California. The views and opinions of authors expressed herein do not necessarily state or reflect those of the United States Government or the University of California, and shall not be used for advertising or product endorsement purposes.

This report has been reproduced directly from the best available copy.

A vailable to DOE and DOE contractors from the Office of Scientific and Technical Information P.O. Box 62, Oak Ridge, TN 37831

Prices available from (615) 576-8401, FTS 626.8401

Available to the public from the National Technical Information Service

US. Department of Commerce 5285 Port Royal Rd. Springfield, VA 22161 


\title{
Continental Seismic Events Observed by the MPL Vertical DIFAR Array
}

\author{
D.B. Harris (LLNL)* \\ G. D'Spain (MPL/SIO)**
}

prepared for DOE/IS20

November 1993

Lawrence Livermore
National Laboratory

* Lawrence Livermore National Laboratory

** Marine Physical Laboratory, Scripps Institute of Oceanography 


\begin{abstract}
The vertical DIFAR array, an underwater acoustic sensor system, deployed by the Marine Physical Laboratory (MPL) was in place over the continental shelf off of Southern California and recorded the HUNTERS TROPHY nuclear ıst and nearly a score of aftershocks of the Landers/Big Bear earthquakes. Data from this array raise the possibility that detection thresholds for continental events may be significantly lower for arrays over the continental shelf than for arrays in the deep ocean basins. Offshore stations could be used to fill gaps in land-based seismic networks for monitoring the NPT and a CTBT, especially for monitoring non-cooperating nations with large coastlines. This preliminary report provides an analysis of the HUNTERS TROPHY observation as well as one of the Landers aftershocks. The analysis suggests detection thresholds for vertical hydrophone arrays below mb 3.0 at ranges between 3 and 4 degrees, and below mib 4.4 out to 6 degrees. This report also describes two signal processing techniques that enhance the detection potential of short vertical arrays. These methods are deterministic null steering to suppress horizontally propagating ambient ocean noise, and matched field processing for vertically-incident acoustic fields. The latter technique is ideally suited for acoustic fields derived from incident seismic waves, and may be viewed as a "synthetic aperture" approach to increase the effective aperture of the array.
\end{abstract}




\subsection{Introduction}

The objective of the Hydroacoustic Proliferation Monitoring project is to determine the feasibility of detecting, locating, and discriminating continental and oceanic nuclear weapon tests using oceanic hydrophone arrays. Our initial focus is on monitoring continental land masses from adjacent oceans. In this context, the value of sensors deployed in the oceans is to fill gaps in land-based seismic coverage, and possibly to provide a rapidresponse monitoring capability.

An initial study of a deep-water observation of the nuclear event LOCKNEY (mb 5.7) suggested a detection threshold in the vicinity of mb 5.2 at a range of $900 \mathrm{~km}$ using a single hydrophone, and perhaps mb 4.2 using a vertical line array [Harris, D'Spain and Goldner, 1993]. That study focused on the use of noise cancellation methods to suppress horizontally propagating ambient noise and sensor noise generated by the array suspension cable. With 22 sensors, $20-25 \mathrm{~dB}$ of rejection was demonstrated against these combined noise sources. The best noise rejection to be expected with spatially incoherent noise is about $13.4 \mathrm{~dB}$ with 22 sensors. Better rejection was achieved in the case of LOCKNEY because the noise was spatially structured, i.e. predominantly horizontal in the case of ambient acoustic noise and downward-travelling in the case of suspension noise. Our signal processing algorithms exploited this structure to suppress the noise. Due to the waveguide nature of sound propagation in the oceans, similar noise structure and processing gains are to be expected generally [Sotorin and Hodgkiss, 1990].

Data available from the Sept. $1992 \mathrm{MPL}$ vertical DIFAR array deployment suggest a much lower detection threshold for vertical arrays deployed over the continental shelf when contrasted with deep-water arrays. Several factors may contribute to an improved detection threshold. First, the array is much closer to continental sources when deployed over the shelf. Signal loss due to geometric spreading and attenuation is correspondingly less. However, the observed increases in signal strength are too large for this factor to explain. Second, the vertical DIFAR array may have been deployed in a "sweet spot" where local focusing effects in the crust or upper mantle act to increase the signal strength. Third, the vertical line array observing LOCKNEY may have been at an especially poor azimuth; there may have been a significant radiation pattern effect that decreased the signal strength for that observation. Finally, continental shelf sites may be especially good for observing continental events. Because the continental structure continues unbroken beneath the shelf, the observed blockage of regional phases for substantial oceanic paths [such as Lg; Ewing, Jardetsky and Press, 1957; Kennett et al., 1985] may not occur.

It is important to resolve these issues, since they have a significant impact on the choice of oceanic monitoring locations (deep water or shallow continental shelf waters), if it is decided to monitor the continents from the oceans. If the observed signal strength of LOCKNEY was uncharacteristically weak due to a source region radiation pattern effect, we have gained no information on optimal array sites. If the "sweet spot" explanation is more likely for the vertical DIFAR observations, then we are left with the undesirable ne- 
cessity of performing a trial and error search for good locations. However, if the continental shelf structural explanation is most likely, we have a clear strategy for array siting.

We will not resolve these issues in this report. To do so will require extensive analysis of many events. However, we will show why these issues are worth considering, by presenting observations of two continental events made at over continental shelf location: the nuclear event HUNTERS TROPHY (mb 4.4, range: $618 \mathrm{~km}$ ), and an mb 3.3 aftershock of the Landers earthquake (range: $322 \mathrm{~km}$ ). For both of these events, a signal was observable on individual hydrophones with processing no more sophisticated than frequency filtering. Especially for HUNTERS TROPHY, this result was not anticipated by the LOCKNEY experience. These observations raise the possibility, yet unproven, that detection thresholds for hydrophone arrays may be below magnitude 3 for events within 3.5 degrees, and below magnitude 4.4 for events within 6 degrees.

Since detection is the first issue facing any newly-proposed monitoring method, we explore at some length various signal processing options that significantly enhance our ability to observe weak signals. In the LOCKNEY study, we demonstrated that noise cancellation may be used to discriminate the upward-propagating acoustic signals generated by seismic waves from the predominantly horizontally-propagating ambient ocean noise. We extend this approach here to a substantially smaller array than was available in that case. In addition, we address the possibility of using still more information about the spatial structure of the seismic-hydroacoustic signal to enhance detection of weak signals. Not only do these waves propagate predominantly vertically in the water column, but they reverberate in a predictable fashion in the oceanic waveguide, reflecting alternately at the free surface and the ocean bottom in a manner that further distinguishes them from the ambient ocean noise.

This is an interim report that raises important monitoring possibilities by demonstrating two new hydroacoustic observations of continental seismic events, as well as additional signal processing potential. In subsequent reports, we will attempt to resolve the questions raised here about the value of continental shelf monitoring sites, using at least a dozen Landers aftershocks observed by the vertical DIFAR array, and data from additional sensor systems.

\subsection{Data}

\subsection{Array Configuration}

The MPL DIFAR array is a vertical line array consisting of 16 sensor packages spaced at 15 meter intervals. Each sensor package consists of a three-component set of geopnones to record acoustic particle motion and a hydrophone to record acoustic pressure. The term DIFAR is an acronym for DIrectional low Erequency Analysis and Recording, and was originally coined for sonobuoys. During the Sept. 1992 deployment, the array was deployed at $32.4 \mathrm{~N}$ and $119.717 \mathrm{~W}$ in approximately 1307 meters of water from a specially stabilized surface vessel (research platform FLIP). Reference to the map of Figure 1 shows that this site is over the continental margin at the edge of the Los Angeles basin. 


\subsection{Landers Aftershock}

A series of aftershocks of the Big Bear / Landers earthquakes occurred during the vertical DIFAR deployment. Data from one of these, a magnitude 3.3 event located at $33.91 \mathrm{~N}$ $116.768 \mathrm{~W}$, are shown in Figure 2. This event was 322 kilometers from the vertical DIFAR array. The acoustic signal has a high signal-to-noise ratio on individual hydrophones. Twelve hydrophones recorded recorded good signals for this event. The spectrogram of one uncalibrated hydrophone record (Figure 3) shows that the signal has energy from at least 2 to $15 \mathrm{~Hz}$. The most remarkable feature of the signal is a pronounced spectral scalloping (a series of nulls in the spectrum spaced at approximately $1 \mathrm{~Hz}$ ). This feature of the spectrum is caused by interference between the upcoming pressure wave, generated by conversion from incident seismic waves at the sea floor, with the downward travelling reflections of these waves. Since the reflection coefficient at the air-water interface is approximately -1 , the modulation of the signal's Fourier transform $s(\omega)$ takes the form:

$$
\left(1-e^{-j \omega T}\right) s(\omega)
$$

where $T$ is the round-trip travel time from the hydrophone to the free surface, then back to the hydrophone. The signal transform has zeroes at intervals of $1 / T \mathrm{~Hz}$.

\subsection{HUNTERS TROPHY}

An acoustic signal from HUNTERS TROPHY is apparent on individual hydrophones at the vertical DIFAR location, at a range of about 618 kilometers from the NTS source. Figure 4 displays the available hydrophone data (12 channels) for a 300 -second interval including the first arrival. The data have been filtered into the 3 to $8 \mathrm{~Hz}$ band to show the arrival, which occurs about 83 seconds into the record. This $P$ phase arrives at about 17:01:26 GMT, which corresponds to a travel time of 86 seconds from the source. The travel time is consistent with a Pn arrival using the Gutenberg formula described in Richter [1958].

The signal from this event has little or no energy above the noise above $10 \mathrm{~Hz}$, a result consistent with the greater path length compared to that of the Landers aftershock. The signal bandwidth is apparent from the spectrogram of Figure 5 generated from the recording of one hydrophone. The signal spectrum exhibits scalloping, though not as clearly as in the previous example.

The mere fact that signals from this relatively small event are observed by individual hydrophones over the continental shelf suggests that some special propagation condition is operating in the vertical DIFAR location that reduces the detection threshold substantially over the VLA deep-water site. In our previous paper on the LOCKNEY observation at the VLA site, we predicted a detection threshold of approximately mb 5.2 for single hydrophones at a range of 900 kilometers for NTS events. Yet an mb 4.4 event is observed well above the noise by a single hydrophone at a range greater than 600 kilometers. Assuming a very low oceanic $\mathrm{Q}\left(Q_{\alpha}=500\right)$ and amplitude loss like $r^{-1}$, the detection threshold would scale to about 4.6. Geometric spreading and intrinsic attenuation do not account entirely for the drop in the detection threshold. We are led to several hypotheses: (1) that the 
continental crust which continues unbroken out under the continental shelf, acts as a waveguide for regional seismic phases, accounting for significant detectable energy, or (2) that the observed strong signal is due to an especially favorable source-region radiation pattern effect or a receiver-region focusing effect.

A decision about which of these explanations is more likely must await analysis of many more events. It is important from a practical standpoint that we know, since the first hypothesis suggests that continental shelf sites may be predictably good locations for monitoring the continents, and the second suggests that good sites will have to be sought by trial and error.

\subsection{Signal Processing for Detection}

The first issue to address from the monitoring perspective is the ultimate detection threshold achievable in an offshore environment. We have the made the casual observation that small events are observable with single hydrophones. However, it is certain that array signal processing methods will substantially reduce the detection threshold. We review and introduce methods to suppress noise that we will use in subsequent reports to estimate the achievable detection threshold.

\subsection{Plane-Wave Beamforming}

The simple noise cancellation approach used to enhance signal to noise ratio with the LOCKNEY data [Harris, D'Spain and Goldner, 1993] cannot be applied directly to the vertical DIFAR array data. In that earlier paper, we observed that the noise field consisted of two principal components: horizontally-propagating ambient acoustic noise and downward propagating tension waves in the array suspension (cable). A suitable processing strategy in that case was to direct a principal beam downward to observe the upward travelling converted seismic signal, and to direct two ancillary beams toward the identified noise sources to use as references in a cancellor. The strategy succeeded since the VLA had a sufficiently large aperture ( 345 meters in the subarray we used) that the principal and ancillary beams were orthogonal. Had the ancillary beams been contaminated with signal, the cancellor would have suppressed the signal along with the noise. The portion of the vertical DIFAR array that yielded usable data (typically 12 out of 16 hydrophones) had an aperture of 165 meters, less than half that of the VLA. In the low frequency range $(5 \mathrm{~Hz})$, the horizontal beam and the downward-directed beam overlap leading to significant signal loss.

Signal cancellation can be avoided by constraining the array response to have nulls in certain pre-determined directions. The null constraints allows us to alter the horizontal and vertical beams to insure they no longer overlap appreciably. The technique results in a deterministic beamformer that performs as well as adaptive processing for the special circumstances of the vertical DIFAR array operated in the 2-12 Hz band at the Sept. 1992 location. 
The procedure for constraining the array response is simple in the frequency domain. We provide some mathematical detail here. The assumption behind most beamforming algorithms (procedures for combining the signals from all array elements to enhance signal and reject noise) is that the incident signal is a single plane wave. Under this assumption, the Fourier transform of the array waveforms have the structure:

$$
r(\omega)=\left[\begin{array}{c}
e^{j\left(\underline{k} \cdot x_{1}\right)} \\
e^{j\left(k \cdot x_{2}\right)} \\
\cdots \\
e^{j\left(\underline{k} \cdot \underline{x}_{N}\right)}
\end{array}\right] s(\omega)
$$

where $r(\omega)$ is the vector of recorded waveforms, the vectors $\underline{x}_{i}$ represent hydrophone locations in the coordinate system (vertical down positive, north, east), the signal has the transform $s(\omega)$, and the direction of approach of the incoming wave is represented in the wavenumber vector:

$$
k=\frac{\omega}{v}\left[\begin{array}{c}
\cos \phi \\
\sin \phi \cos \theta \\
\sin \phi \sin \theta
\end{array}\right]
$$

where $\phi$ is the angle of incidence of the wave (from vertical), $\theta$ is the receiver-source bearing, $\omega$ is the frequency (radians/sec) and $v$ is the medium velocity. The vector of phase delays in equation 2 , which we denote by $\underline{e}(\phi, \theta, \omega)$ is commonly called the array steering vector.

The beamforming operation may be stated succinctly in the frequency domain. Beamforming consists of combining the vector of recorded waveforms to enhance signals from a particular direction. A weight vector $\underline{w}$ is used to construct a scalar signal:

$$
b(\omega)=\underline{w}^{H} r(\omega)
$$

where the superscript $H$ denotes the conjugate transpose operation. The conventional beam is obtained by selecting:

$$
\underline{w}=\left(\underline{e}^{H} \underline{e}\right)^{-1} \underline{e}
$$

which recovers the signal (i.e. $b(\omega)=s(\omega)$ ) if the direction of the beamformer is chosen appropriately. Note that we can specify the design of the beamformer $\underline{w}$ by constraining its response to the appropriate plane wave $(\underline{e})$ to be one: $\underline{w}^{H} \underline{e}=1$.

Additional constraints can be applied to ensure that the beamformer has no response in other directions. By applying null constraints of the form $\underline{w}^{H} \underline{e}_{i}=0$, we ensure that noise from these other specified directions is suppressed. We can express the collection of con- 
straints in the signal direction (also called the look direction) and in the null directions in a matrix form suitable for computations:

$$
{ }^{H}{ }^{H} C=f \quad C=\left|\begin{array}{llll}
e & e_{1} & \ldots & e_{p}
\end{array}\right| \quad f=\left|\begin{array}{llll}
1 & 0 & \ldots & 0
\end{array}\right|
$$

The beamformer is calculated using the expression:

$$
\underline{w}=\underline{C}\left(\underline{C}^{H} C\right)^{-1} f^{T}
$$

Figure 6 demonstrates the practical application of these expressions to the problem of detecting upcoming waves in the presence of noise and reflections from the surface. The figure displays polar plots of the vertical DIFAR array response (i.e. $\left.\left|\underline{w}^{H} \underline{e}\right|\right)$ for all angles of incidence in the vertical plane evaluated for a frequency of $5 \mathrm{~Hz}$. Since the vertical DIFAR is a line array, the response is uniform in all bearings; the complete three-dimensional array responses are figures of rotation of the displayed cross-sections about the vertical axis.

The conventional beam directed straight down (to detect upcoming waves) is shown in Figure 6a. At this low frequency, the array is roughly one-half wavelength long, and has very little resolution. The array has a significant response to horizontally-propagating waves, as well as a smaller response to waves travelling down from the surface. An attempt to reduce sensitivity to waves travelling vertically downward is shown in Figure $6 \mathrm{~b}$. This is the response for a beamformer with a look-direction constraint in the downward direction and a single null constraint in the upward direction. This beam succeeds in eliminating downward-travelling waves, but has virtually the same response to horizontally travelling waves.

Horizontally travelling waves may be suppressed by placing a null in the horizontal direction, as shown in Figure $6 c$. The response is much improved in the horizontal direction, but has a very large lobe projecting upwards. Figure $6 \mathrm{~d}$ shows the result of directing a null upward to correct this lobe. This beamformer not only separates upgoing waves from downgoing waves well, but also has a narrower main lobe in the downlooking direction. Consequently it should be better at rejecting horizontally travelling noise as well.

Figures 7 and 8 demonstrate that the beamformer characteristics just described are realized when data are processed. Figure 7 shows the four beams corresponding to the four beamformers when applied to the Landers aftershock. To implement the beamformer, special software was developed to break the wideband signal into narrowband (essentially single-frequency) components, to form the beams on each narrowband component, then to reassemble the components into the wideband beam. The first trace shows the simple downward-directed beam; the beam has been clipped to make the background noise visible. The second trace is the beam from the processor with a null directed upward: it looksvery similar to the first beam. The bottom two beams show significant reductions in the background noise. The third trace is due to the beamformer with the single, horizontal null. A significant decrease in background noise is apparent (about $6.5 \mathrm{~dB}$ ) over the conventional beam result in the $3-7 \mathrm{~Hz}$ band. The bottom trace, which corresponds to the 
beamformer with nulls directed horizontally and in the upward direction is still better with $8.4 \mathrm{~dB}$ less noise than the conventional downward-looking beam.

Figure 8 demonstrates the value of vertical nulls for separating upgoing and downgoing waves. It displays 20 -second windows of the downlooking beam with uplooking and horizontal nulls, and four comparable uplooking beams; a conventional beam directed upwards, an uplooking beam with a horizontal null, an uplooking beam with a downwarddirected null, and an uplooking beam with both horizontal and downward-directed nulls. The first two uplooking beams are cciitaminated by the initial upcoming converted $P$ phase. However, the placement of a null in the downlooking direction effectively eliminates the contamination. By comparing the downlooking beam (top) with the upcoming beam (bottom), the reflection of the incident pulse at the free surface, and the two-way propagation delay time ( 0.93 seconds) is obvious. This type of processing is important for understanding the reverberant signal structure. By decomposing the wavefield into its downgoing and upgoing components, we increase the potential for more sophisticated detection and discrimination algorithms.

\subsection{Matched Field Processing}

The observation that seismic signais refract vertically into the water column, then reverberate by reflecting alternately from the free surface and the ocean bottom (Figure 9) leads to an additional signal processing strategy for suppressing noise. Instead of using simple beamforming to pass an incoming plane wave, we design the detector to pass the entire reverberant structure of the seismo-acoustic signal. This approach is called matched field processing (MFP) and requires a more ccmprehensive model for the incident wave field. In principal, MFP is better able to reject noise by being more specific about the spatial structure of the signal that it is designed to detect. The term matched field refers to the fact that the processor acts as a spatial and temporal correlator (matched filter) for the specified signal structure.

The matched field processor for seismic arrivals that convert to near-vertically travelling acoustic waves in a uniform water column has a particularly simple structure. As is shown in detail in the Appendix, the signal model factors into the product of a spatially-varying component that depends only upon free-surface interaction and a spatially-invariant component that depends on both free-surface and bottom reflections (the round-trip from the phone to the free surface, down to the bottom, and back up to the phone). This fact leads to a matched field processor that is a cascade of two pieces. The first part is a beamformer designed to pass two interacting waves: a wave incident from below the array combined with its free-surface reflection. The input to this processor is the multichannel waveform from the array, and its output is a single (scalar) beam waveform. The second part is a scalar matched filter for the reverberant signal due to the full acoustic round-trip path between the surface and the bottom.

This decomposition of the processor is a significant simplification because the most complicated component, the front-end beamformer, is not a function of bottom reflection characteristics or of the water depth. It depends solely on the array configuration and the direction of the refracted acoustic waves. The scalar matched filter at the back-end of the 
processor depends only on the water depth, the reflection coefficient at the bottom, and the round-trip travel time for a near-vertical internally-reflected wave in the water column. This part of the processor does not depend on the detailed structure of the array, nor upon the depth of the array.

A further simplification is possible for short arrays. If the array is not too deep and less than a wavelength long, one can assume that the acoustic fields propagate exactly vertically across the array aperture. As seen in Figure 6, the vertical DIFAR array is insensitive to variations in the incidence angle within about 20 degrees from vertical. Consequently, the front-end beamformer need be designed only for exact vertical incidence. It must pass two signals: one upcoming which is inverted, and the second downgoing, which is delayed by the two-way travel time from the array to the surface and back. The structure of the simple processor is shown in Figure 10. Note that the "beamformer" front end consists of separate downlooking and an uplooking beamformers. It is possible to take advantage of null placement to suppress horizontal noise as described in the previous section. In the examples that follow, we take advantage of this fact.

The front-end beamformer can be viewed as implementing a synthetic aperture array. With short arrays, resolution is a problem that may be alleviated by exploiting the nearperfect reflector at the ocean surface. By combining the upgoing and downgoing reflected waves, we are effectively incorporating virtual sensors imaged about the free surface. The synthetic aperture is equal to twice the depth of the array, which is much larger than the physical array aperture. While some noise rejection is achieved by this synthetic aperture processing, far less is achieved than would be with a physical aperture of the same dimensions. The problem with this processing approach is that it emulates an extremely sparse array, with all of its sensors concentrated at the extremities of the synthetic aperture. The resolution of the processing is enormously increased at the cost of very poor sidelobe structure. The best use of the synthetic aperture principal may be in seismic phase identification, i.e. in measuring the relatively small variations in angle of incidence of different seismic waves refracting up into the water column.

To date, we have implemented just the front-end beanforming section of the matched field processor, but it alone is sufficient to increase slightly the signal-to-noise ratio for detection over the null-constrained beams. This fact is demonstrated by applying the frontend of the matched field processor to the HUNTERS TROPHY data. Figure 11 shows the result of beamforming these data with three alternative processors in the 3 to $7 \mathrm{~Hz}$ band. In this example, we contrast the signal from an individual hydrophone, the simple downlooking beam, the simple beam with nulls in the horizontal and uplooking direction, and the matched field processor. To examine how much gain is attained by these different processing options, we defined a simple measure of signal-to-noise ratio (SNR). Our SNR is the ratio of the energy of the signal in a 20 -second window starting at the signal onset and the energy in a 20 -second ambient noise sample prior to the signal arrival. The measured SNR for the individual hydrophone was $10.4 \mathrm{~dB}$. The simple downlooking beam did not improve this figure much; its SNR was just $12.8 \mathrm{~dB}$. The null-constrained beam had an SNR of $19.9 \mathrm{~dB}$, which is a significant improvement. The matched field processor in this case had an SNR of $21.1 \mathrm{~dB}$. 
At higher frequency, the different modes of beamforming are more nearly equal. Figure 12 shows the same waveforms filtered into the 6 to $10 \mathrm{~Hz}$ band. In this case, the individual hydrophone SNR drops to $6.8 \mathrm{~dB}$, but the simple downlooking beam increases its SNR to $16.3 \mathrm{~dB}$. The null-constrained beam has an SNR of $17.2 \mathrm{~dB}$, and the matched field processor has an SNR of $18.5 \mathrm{~dB}$. As the frequency increases, so do both the effective array aperture and the resolution of the simple processor.

The performance improvement of the matched field processor for these examples is minimal against the ambient acoustic noise, but somewhat better against the transient signals that occur late in the HUNTERS TROPHY data record. The origin of these transients is unknown, but they apparently do not fit the reverberant signal model and are suppressed by the processor. Several effects may contribute to the minimal additional rejection of the ambient acoustic noise. First, the matched field processor would be most effective against horizontally propagating noise, which is already suppressed by the null-constrained beams. Second, the matched field processor was not spatially adaptive in this implementation; an adaptive processor could do more to distinguish structured noise from the reverberant signal. Third, only the front end of the matched field processor was implemented; the inverse channel filter, if implemented, might provide additional noise suppression.

One can argue that the maximum gain afforded by this non-adaptive matched field processor over the null-constrained beams would be $3 \mathrm{~dB}$. The matched field processor delays the downlooking beam, inverts the uplooking beam, and adds the two together. The delay time is large enough to allow the noise to decorrelate, if it is wideband. If the up- and down-going signals add coherently, and the noise incoherently, then the SNR would increase by $3 \mathrm{~dB}$. If the noise is narrowband, the non-adaptive processing gain will be less than $3 \mathrm{~dB}$.

\subsection{Conclusions and Further Work}

From the examples presented in this report, it is apparent that small (probably $<\mathrm{mb} 3.0$ ) continental seismic events may be observed with vertical line arrays at ranges between 3 and 4 degrees from some locations on the continental shelves. In a fairly suprising result, the magnitude 4.4 nuclear test HUNTERS TROPHY was observed with a post-processing SNR of about $20 \mathrm{~dB}$ at a range of 618 kilometers. The remaining questions to be explored in this project concern the generality of these observations.

Superficially, these observations suggest that hydrophone arrays deployed over continental shelves may have significantly lower detection thresholds than arrays deployed in deep-water sites. However, some care must be taken in drawing this conclusion until a significant number of continental event observations are available. As mentioned in the introduction, the relatively low SNR, deep-water LOCKNEY observation could have been an artifact of a source-region radiation pattern effect. This hypothesis could be tested with land-based observations of both LOCKNEY and HUNTERS TROPHY along the great circle paths to the VLA and vertical DIFAR array locations. Similarly, the vertical DIFAR observations could be anomalously strong due to focusing effects in the crust and mantle beneath that array site. Such variations have been documented before, particularly for 
teleseisms observed at the NORSAR subarrays [Ringdal, 1990]. This hypothesis can be examined by analyzing many more events observed by the vertical DIFAR array during its September 1992 deployment (mainly Landers aftershocks), to see if there is a strong azimuthal dependency (after source radiation patterns are accounted for) in signal amplitude. Strong azimuthal dependence would suggest that focusing is taking place. Similarly, observations of the Non-Proliferation Experiment (NPE) made at different locations on the southern California continental margin (including the Sept. 1992 vertical DIFAR location) would test the focusing hypothesis, as would comparisons to USGS station observations of HUNTERS TROPHY in the Los Angeles basin. The immediate need is to process more events and to estimate actual hydroacoustic signal strength.

If the radiation pattern and focusing hypotheses are negative, the case for the continental shelf as a wave guide for regional continental seismic phases is strengthened. As remarked in the introduction, this conclusion would have important implications for siting hydroacoustic systems worldwide for continental monitoring. The plausibility of this continental waveguide hypothesis may be tested further by computing synthetic hydroacoustic observations using our best estimates for crustal and upper mantle structure at the continental margins.

The signal processing methods demonstrated in this report have wider application than just the continental monitoring problem. If free-drifting buoy systems with suspended vertical line arrays were deployed to monitor remote oceanic regions for detonations at sea, the null-constrained and matched field processors described here are simple enough to be implemented with on-board processors to detect seismic as well as acoustic signals. The ability to use free-drifting arrays as seismometers could be very useful for discriminating detections of undersea earthquakes and explosions at sea.

\subsection{Acknowledgements}

Work performed under the auspices of the U.S. Department of Energy by the Lawrence Livermore National Laboratory under Contract W-7405-Eng-48.

The collection of the vertical DIFAR array data was sponsored by the Office of Naval Technology.

\subsection{References}

Ewing, M., W. Jardetsky and F. Press (1957), Elastic Waves in Layer Media, McGrawHill, New York.

Harris, D., G. D'Spain and A. Goldner (1993), "Regional observation of a nuclear test from a vertical hydrophone array," submitted to the Bull. of the Seism. Soc. of Am., LLNL report UCRL-JC-112849. 
Kennett, B., S. Gregersen, S. Mykkeltveit, and S. Newmark, " Guided wave propagation in laterally varying media - II. $L g$ waves in north-western Europe," Geophys. Jour. of the R. Astr. Soc., 79, 257-267.

Richter, C. (1958), Elementary Seismology, W. H. Freeman and Co., San Francisco, p. 686.

Ringdal, F. (1990), "Teleseismic event detection using the NORESS array, with special reference to low-yield Semipalatinsk explosions," Bull. of the Seism. Soc. of Am., 80(6), 2127-2142.

Sotirin, B. and W. Hodgkiss (1990), "Fine-scale measurements of the vertical ambient noise field," Jour. of the Acoustical Soc. of Am., 87(3), 2052-2063.

\subsection{Appendix - Signal Model in a Uniform Medium}

The signal due to an incident (compressional) seismic wave recorded by hydrophone $k$ in an array in the middle of the water column consists of a direct arrival, which we denote by $r_{k}(\omega)$, and an infinite sum of reflections from the free surface and the bottom. We model the direct arrival in a uniform acoustic medium by:

$$
r_{k}(\omega)=e^{j \frac{\omega}{v^{\prime}} d_{k}} r(\omega)
$$

where $d_{k}$ is the sensor depth, $r(\omega)$ is the direct arrival at the surface, and $v^{\prime}=v / \cos \phi$ is the effective vertical velocity in the medium for a wave travelling at incidence angle $\phi$ from vertical. The free surface reflection of this signal is an inverted and delayed version of the signal:

$$
F_{k}(\omega) r_{k}(\omega)=-1 \cdot e^{-j \frac{\omega}{v^{2}} 2 d_{k}} r_{k}(\omega)
$$

where the time delay $2 d_{k} / v^{\prime}$ is the round-trip acoustic travel time from sensor $k$ to the surface and back. Similarly, we model propagation to the bottom and reflection there by the operator:

$$
B_{k}(\omega)=e^{-j \frac{\omega}{v^{\prime}} 2\left(d-d_{k}\right)} R(\omega)
$$

where $d$ is the depth of the water column, and $R(\omega)$ is the bottom reflection coefficient.

The total received signal $y_{k}(\omega)$ at sensor $k$ is the direct arrival superimposed on the infinite series of alternating reflections from the free surface and the bottom. It has the form: 


$$
\begin{gathered}
y_{k}(\omega)=r_{k}(\omega)+F_{k}(\omega) r_{k}(\omega)+B_{k}(\omega) F_{k}(\omega) r_{k}(\omega)+ \\
F_{k}(\omega) B_{k}(\omega) F_{k}(\omega) r_{k}(\omega)+\ldots
\end{gathered}
$$

The terms can be grouped as follows:

$$
y_{k}(\omega)=\left[1+F_{k}(\omega)\right] r_{k}(\omega)+F_{k}(\omega) B_{k}(\omega)\left[1+F_{k}(\omega)\right] r_{k}(\omega)+\ldots
$$

to yield the infinite sum:

$$
y_{k}(\omega)=\sum_{i=0}^{\infty}\left(F_{k}(\omega) B_{k}(\omega)\right)^{i}\left[1+F_{k}(\omega)\right] r_{k}(\omega)
$$

which may be evaluated in closed form:

$$
y_{k}(\omega)=\frac{\left[1+F_{k}(\omega)\right]}{1-F_{k}(\omega) B_{k}(\omega)} r_{k}(\omega)
$$

However, the product of the free surface and bottom operators is independent of the phone position:

$$
F_{k}(\omega) B_{k}(\omega)=-1 \cdot e^{-j \frac{\omega}{v^{\prime}} 2 d_{k}} e^{-j \frac{\omega}{v^{\prime}} 2\left(d-d_{k}\right)} R(\omega)=-R(\omega) e^{-j \frac{\omega}{v^{\prime}} 2 d}=T(\omega) \quad \text { (EQ 15) }
$$

so that the total received signal factors into a term involving the round-trip travel time from the surface to the bottom and back (the channel response), multiplied by phone-specific response involving only interaction with the free surface:

$$
y_{k}(\omega)=\frac{1}{1-T(\omega)} \bullet\left[1+F_{k}(\omega)\right] r_{k}(\omega)
$$

The vector response of the array, then is the product of a scalar bottom-dependent channel reponse term and a vector array response term: 


$$
y(\omega)=\frac{1}{1-T(\omega)} \cdot\left[\begin{array}{c}
{\left[1+F_{1}(\omega)\right] e^{j \omega}} \\
\cdots \\
{\left[1+F_{N}(\omega)\right] e^{j}}
\end{array}\right] r(\omega)
$$

This fact permits us to decouple the matched field processor into a spatial beamformer for the direct path and free-surface interaction, followed by a simple matched filter for the channel response of the water column. 


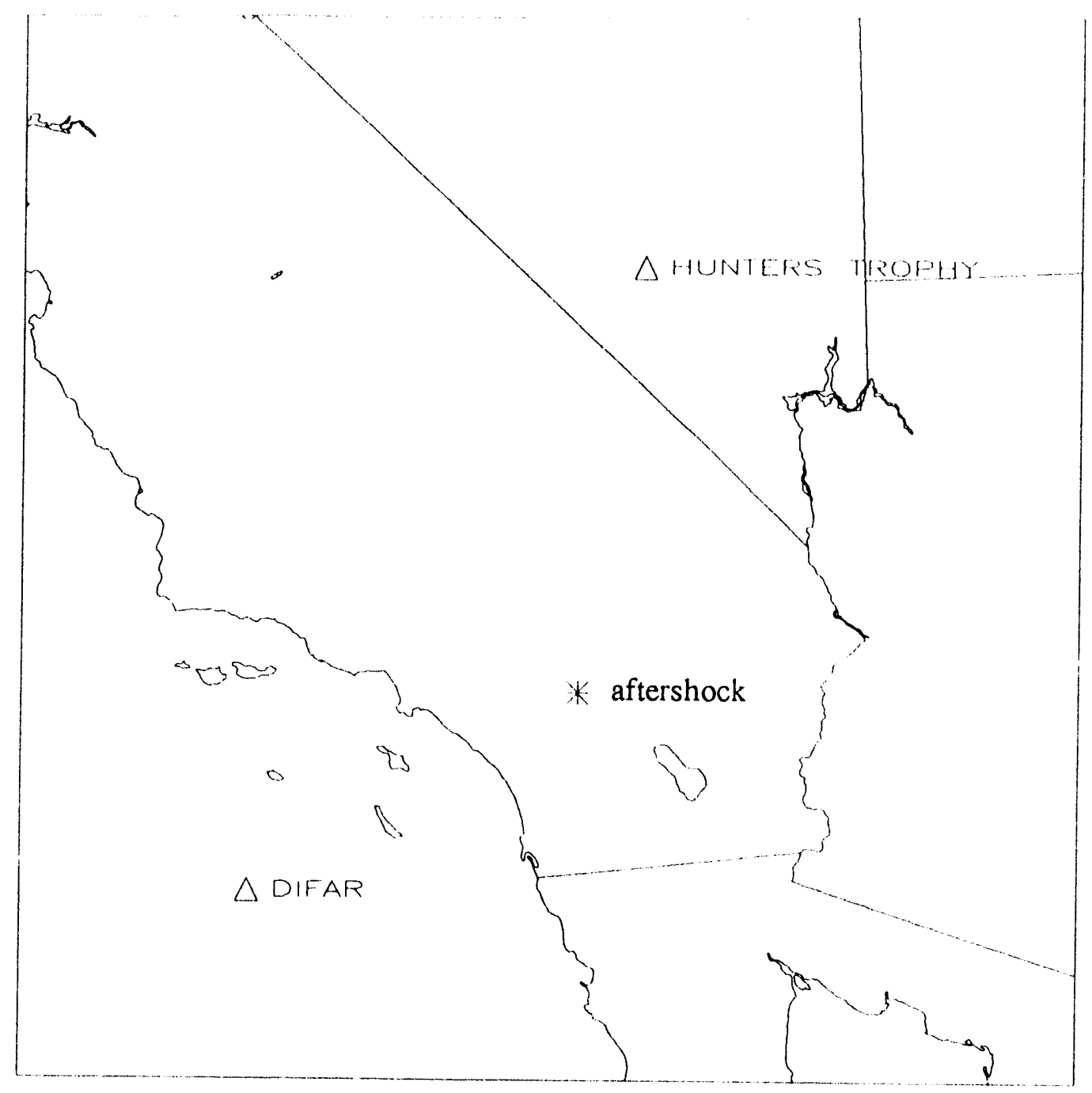

Figure 1 Location of the vertical DIFAR array, the nuclear test HUNTERS TROPHY, and the Landers earthquake aftershock. The vertical DIFAR array was located on the edge of the continental shelf in approximately 1300 meters of water. 


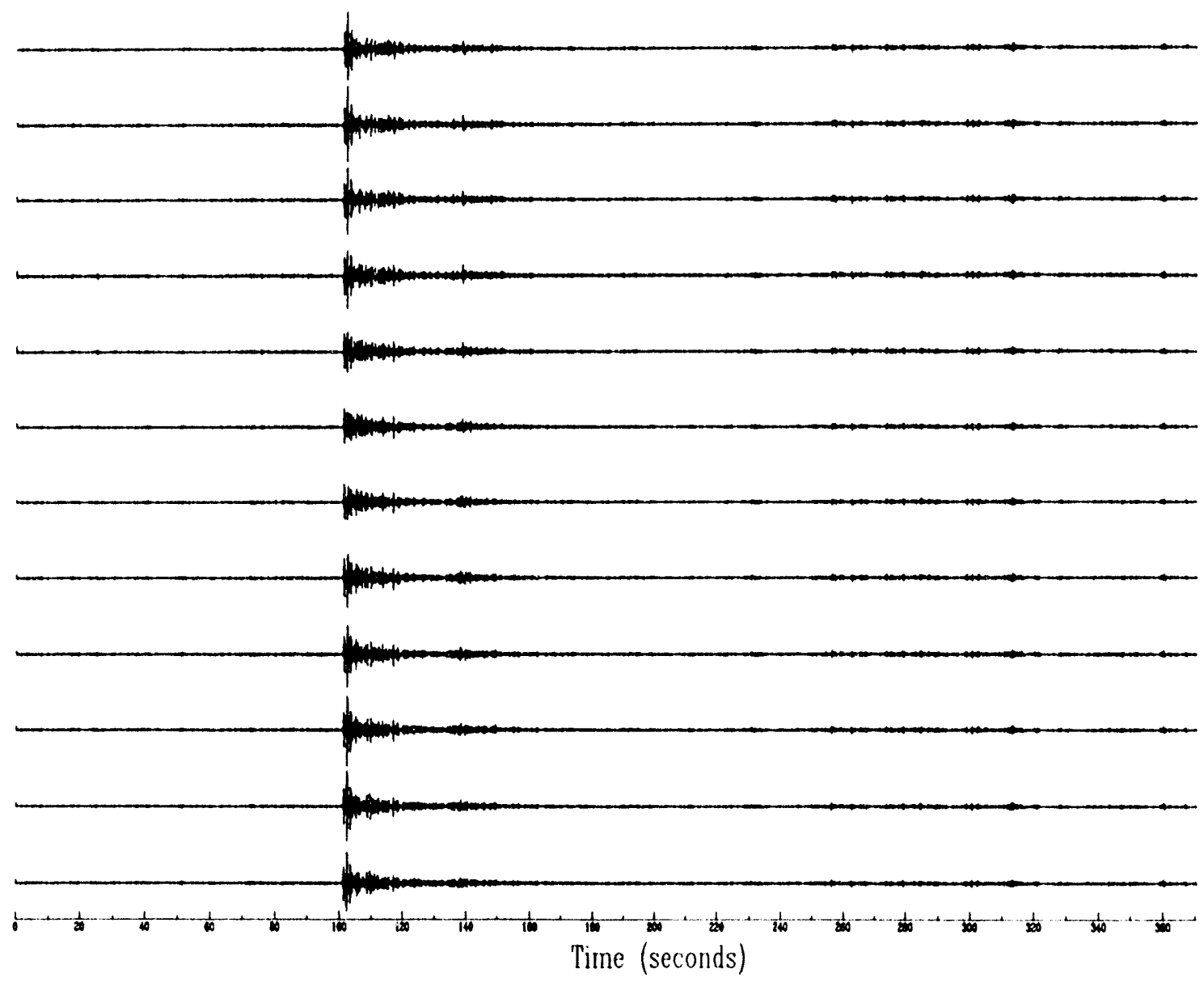

Figure 2 Filtered hydrophone data from the vertical DIFAR array for an mb 3.3 aftershock of the Landers earthquake. The data have been filtered into the 4 to $10 \mathrm{~Hz}$ band, but are otherwise unprocessed. 


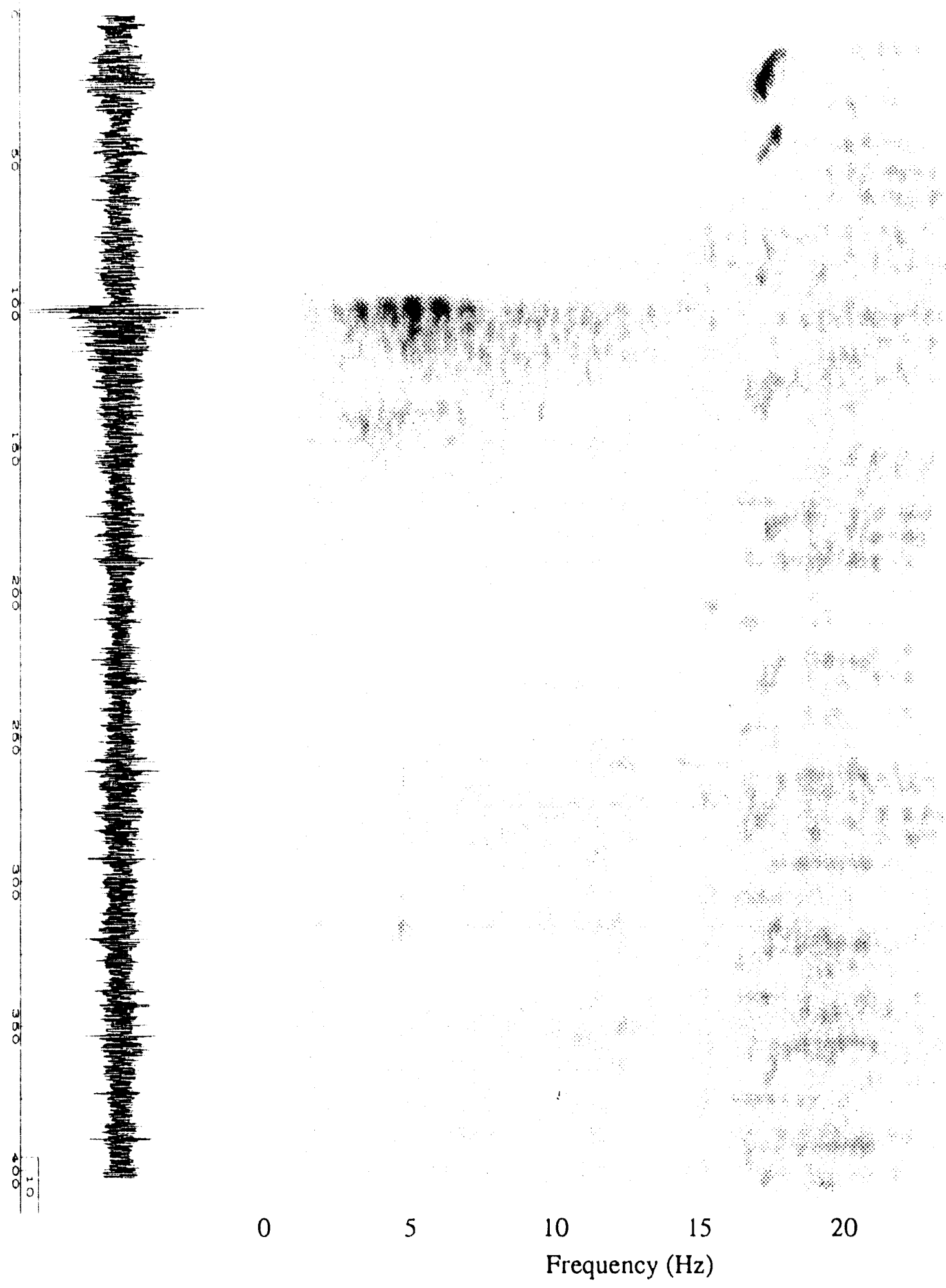

Figure 3 Spectrogram of the Landers aftershock which shows the presence of signal energy to $15 \mathrm{~Hz}$, and pronounced spectral scalloping caused by reverberation in the water column. Time increases from top to bottom in this display (about 400 seconds total), and frequency increases from left to right in the spectrogram. A single hydrophone trace is shown to the left for reference. As often happens, a blue whale contributed the distinctive 20 -sec downchirp visible at $17 \mathrm{~Hz}$ near the top of the record, and more faintly throughout the record. 


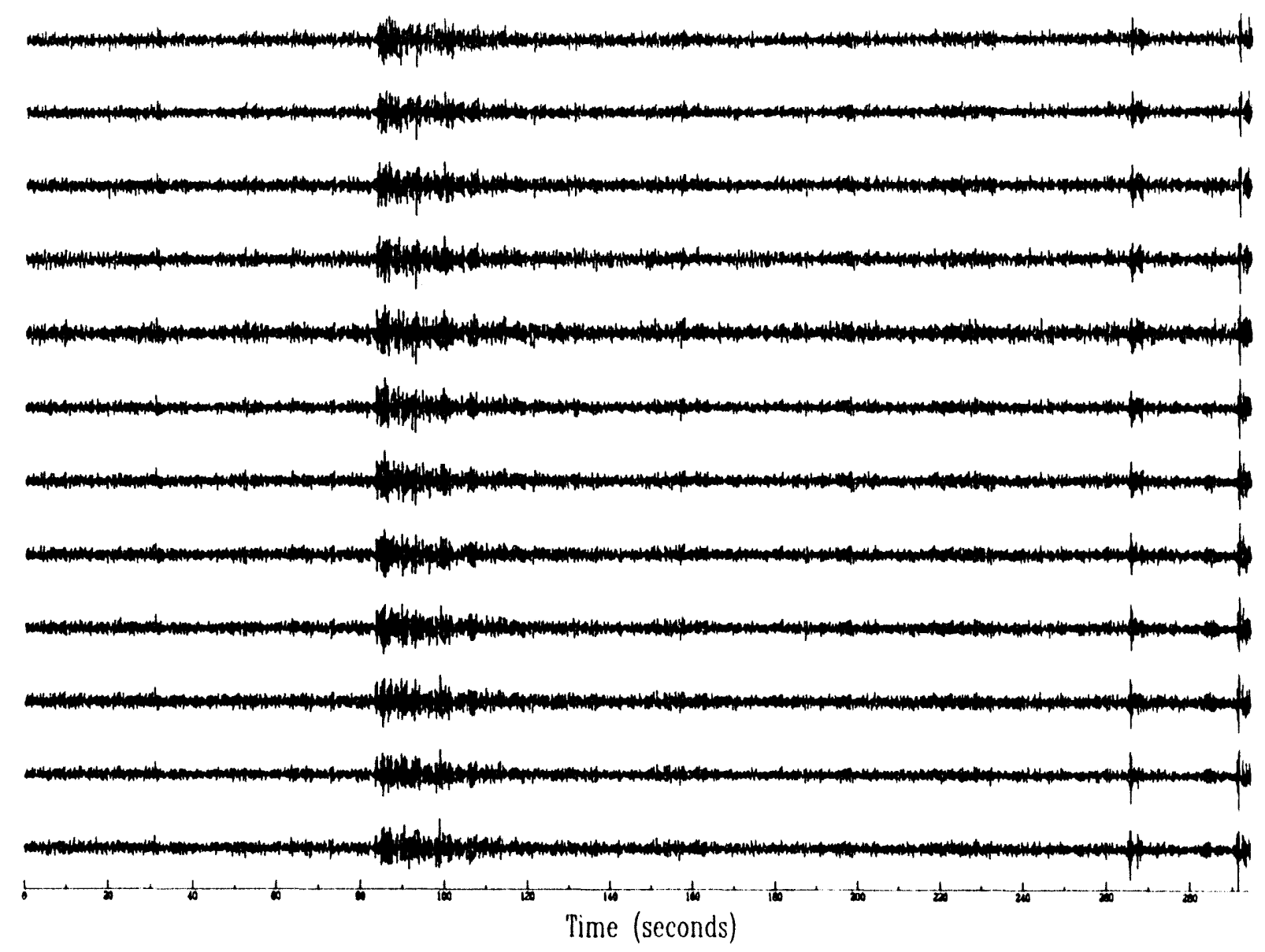

Figure 4 Filtered hydrophone data from the vertical DIFAR array for the nuclear event HUNTERS TROPHY. The data have been filtered into the 3 to $8 \mathrm{~Hz}$ band. The range to the event from the array was 618 kilometers, and the event had a network P wave magnitude of 4.4 . 

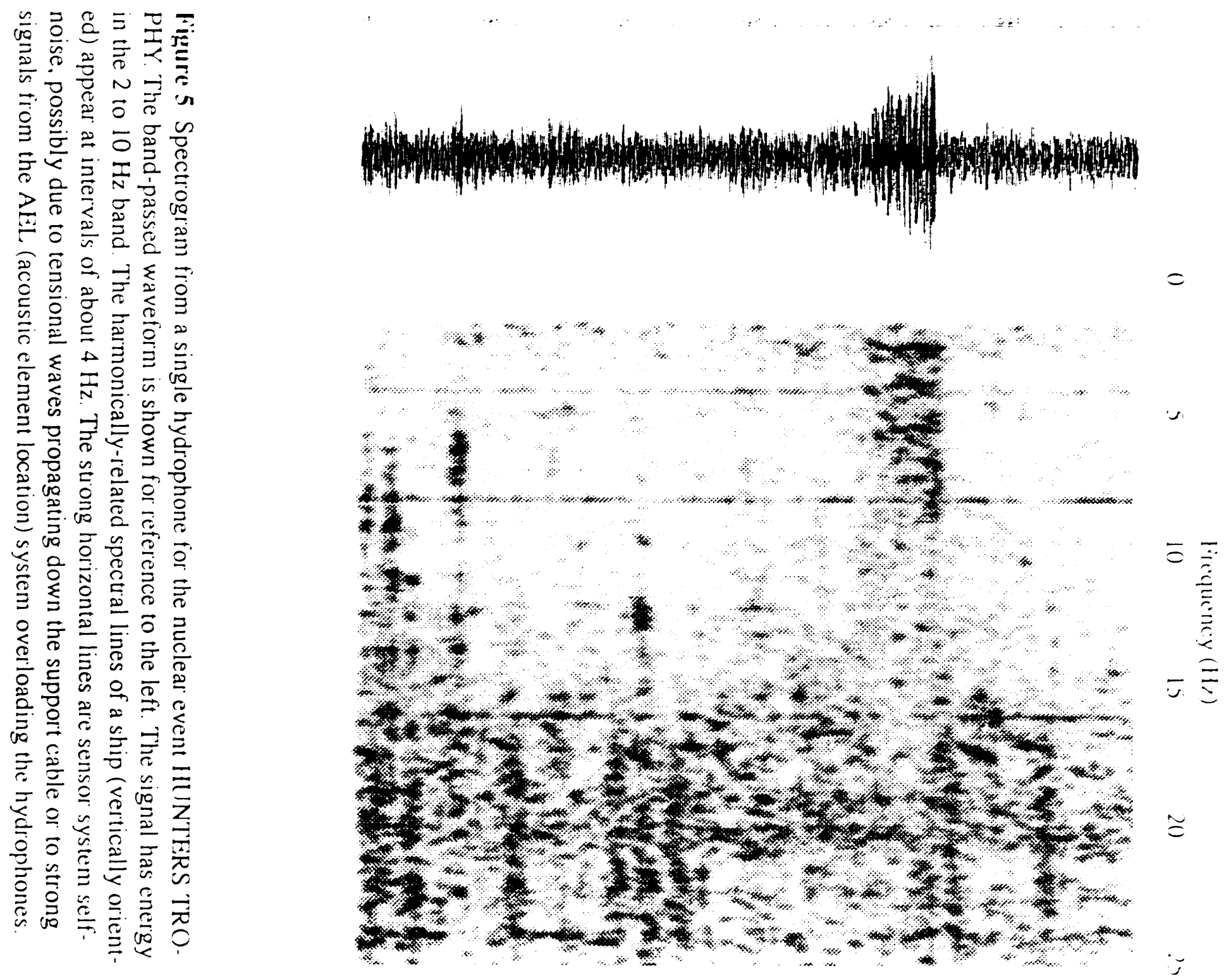
(a)

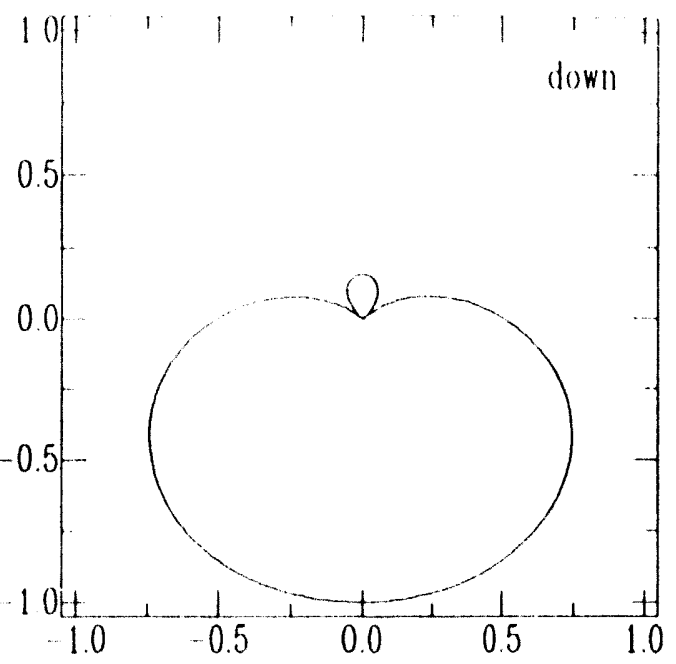

(c)

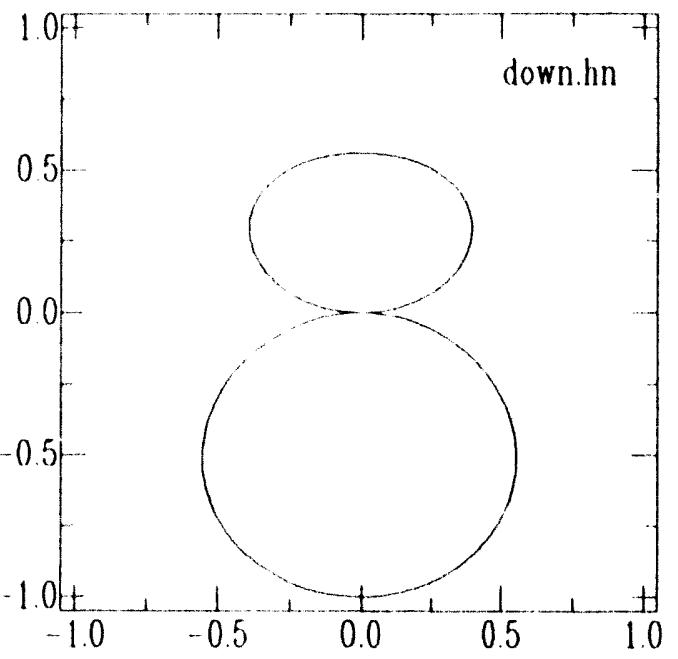

(b)

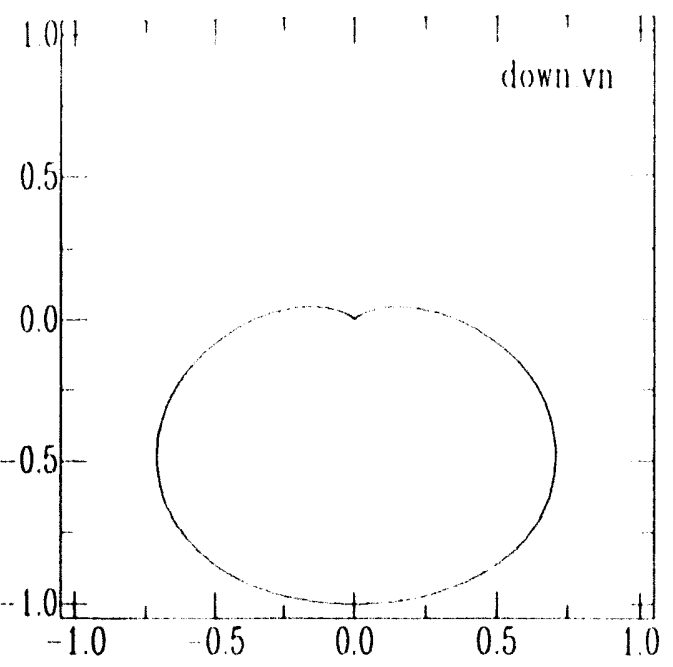

(d)

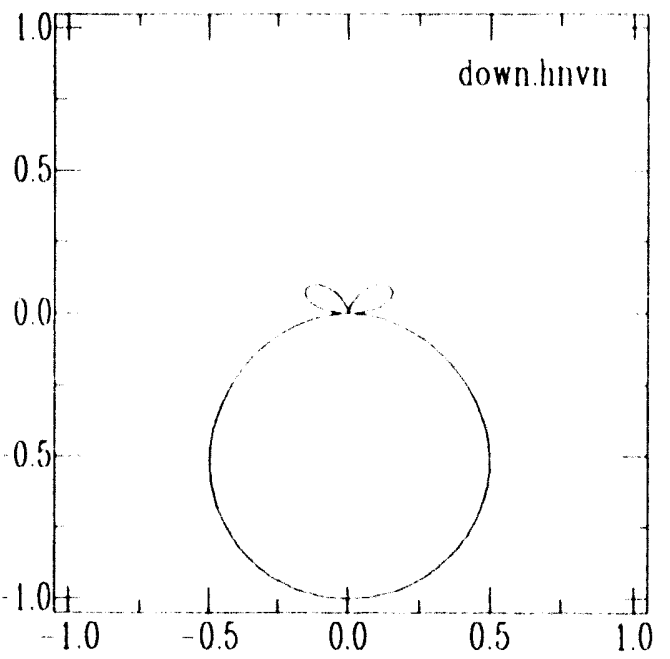

Figure 6 Array amplitude response patterns for four beams indicate that the best performance for separating upgoing and downgoing waves and for suppressing horizontal noise is achieved by a beam that has 3 constraints: ? unit gain constraint directed downward, and two nulls, one directed horizontally and the orher directed upward. These array patterns indicate array response to plane waves as a function of direction at $5 \mathrm{~Hz}$. At this low frequency, endfire array response is insensitive to deviations from exact endfire propagation. The beams are: (a) conventional - look constraint down, (b) look constraint and one null directed toward the surface, (c) look constraint and one null directed horizontally, (d) look constraint with nulls directed horizontally and toward the surface. 


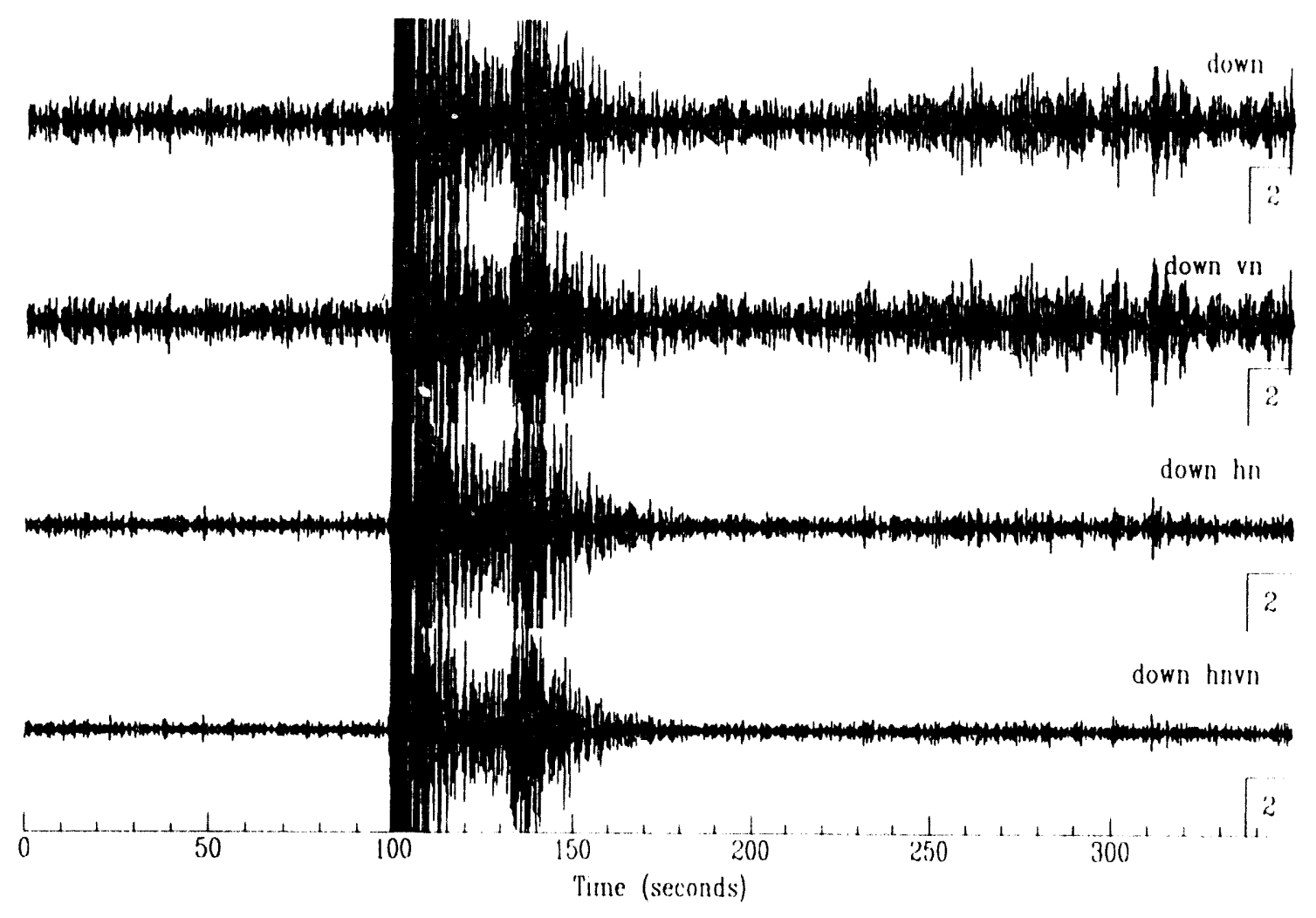

Figure 7 Added null constraints dramatically reduce background noise. Four beams are shown in this figure, corresponding to the array response patterns of Figure 6 . The signals are filtered into the 3-7 Hz band, and intentionally clipped in the display to show the relative amplitudes of background noise. The top beam is a simple downward-directed beam with a single constraint in the look direction (passes an upcoming plane wave). The remaining three beams have additional nulls directed (b) up, (c) horizontally, and (d) both up and horizontally. Since most ambient noise is horizontally propagating, the horizontal nulls do most to reject noise. The combination of nulls in the upward and horizontal directions is best, since the array response pattern in this case is narrowest in its main lobe 


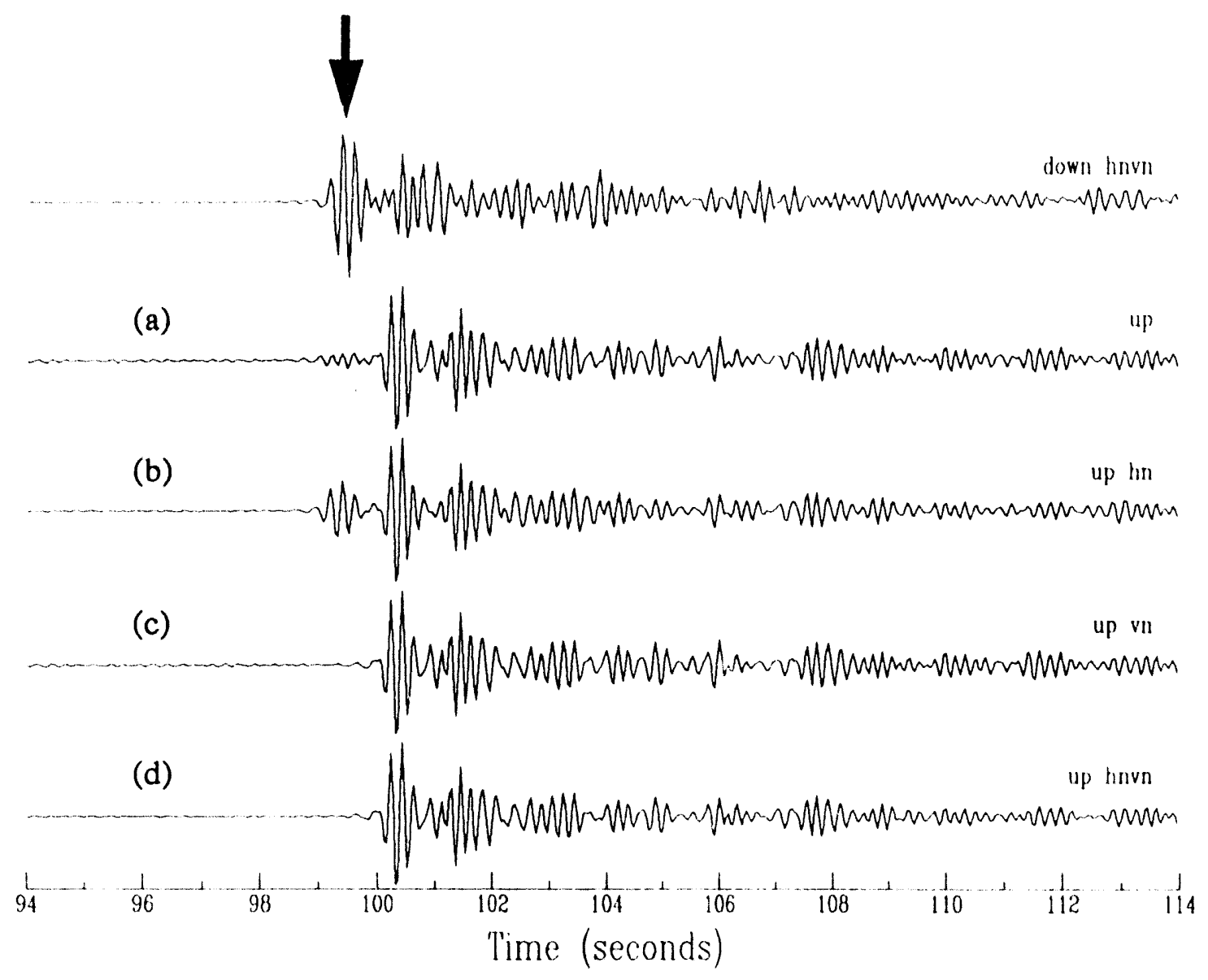

Figure 8 The vertical null constraint is essential for separating upgoing and downgoing waves. The top trace shows the downward-directed beam (passing upcoming waves). Four upward-directed beams are shown below it; these pass waves reflected from the free surface that travel downward past the array. The simple beam (a) and the beam with horizontal null (b) are significantly contaminated by the initial upcoming wave converted at the sea floor (arrow). Beams with downward-directed vertical nulls ( vertical null only (c), horizontal and vertical null (d) ) eliminate the upcoming waves. All data have been filtered into a $3-7 \mathrm{~Hz}$ passband. 


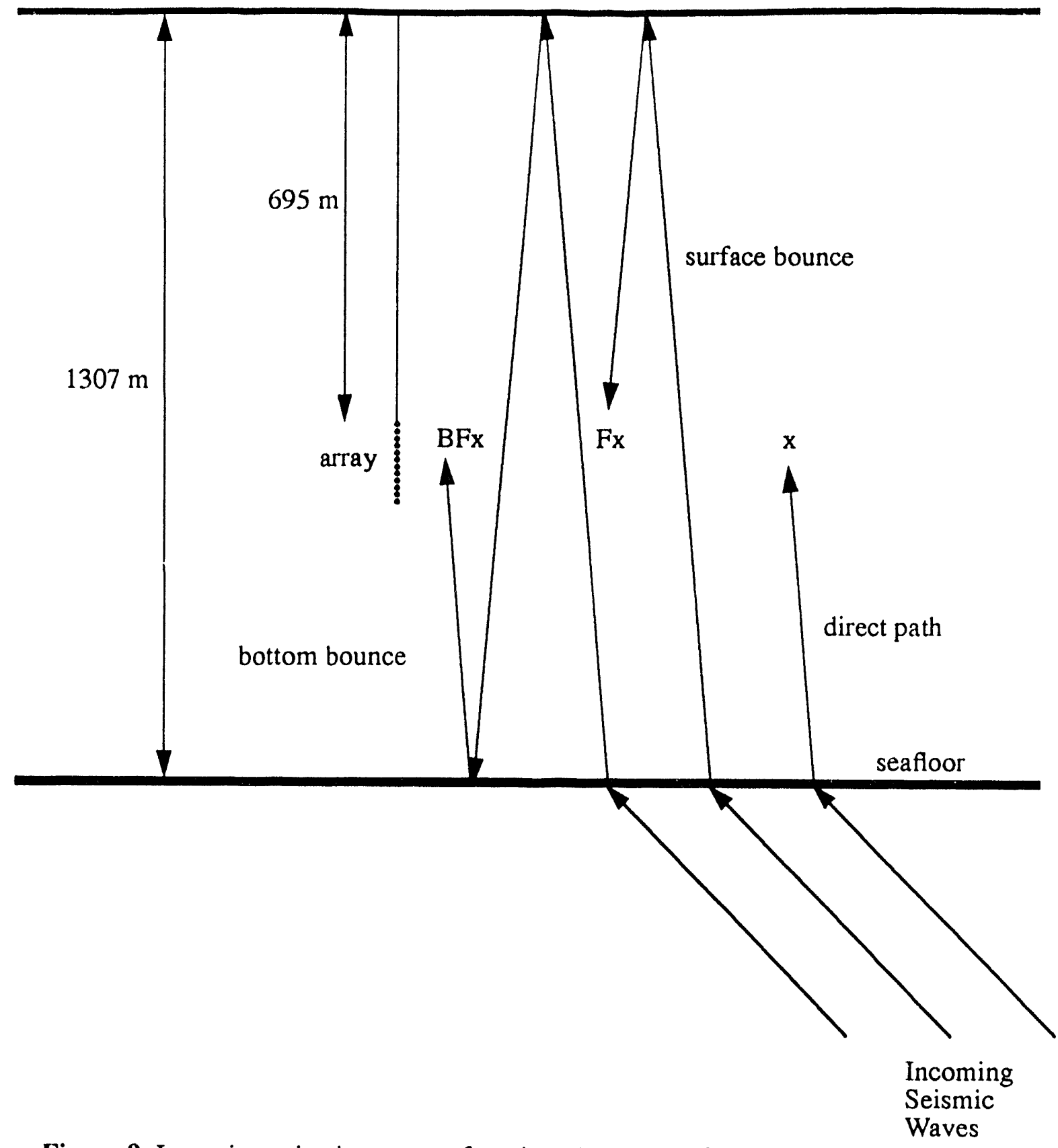

Figure 9 Incoming seismic waves refract into the water column, propagating nearly vertically. In an environment with a perfectly flat sea floor, the signal will reverberate in a predictable fashion reflecting alternately from the free surface and from the bottom. Such reverberation imparts a spatial and temporal structure to the signal that is very unlike the dominant noise: horizontally-propagating noise from ships and storms at sea. This structural difference may be used to suppress noise to some extent. 


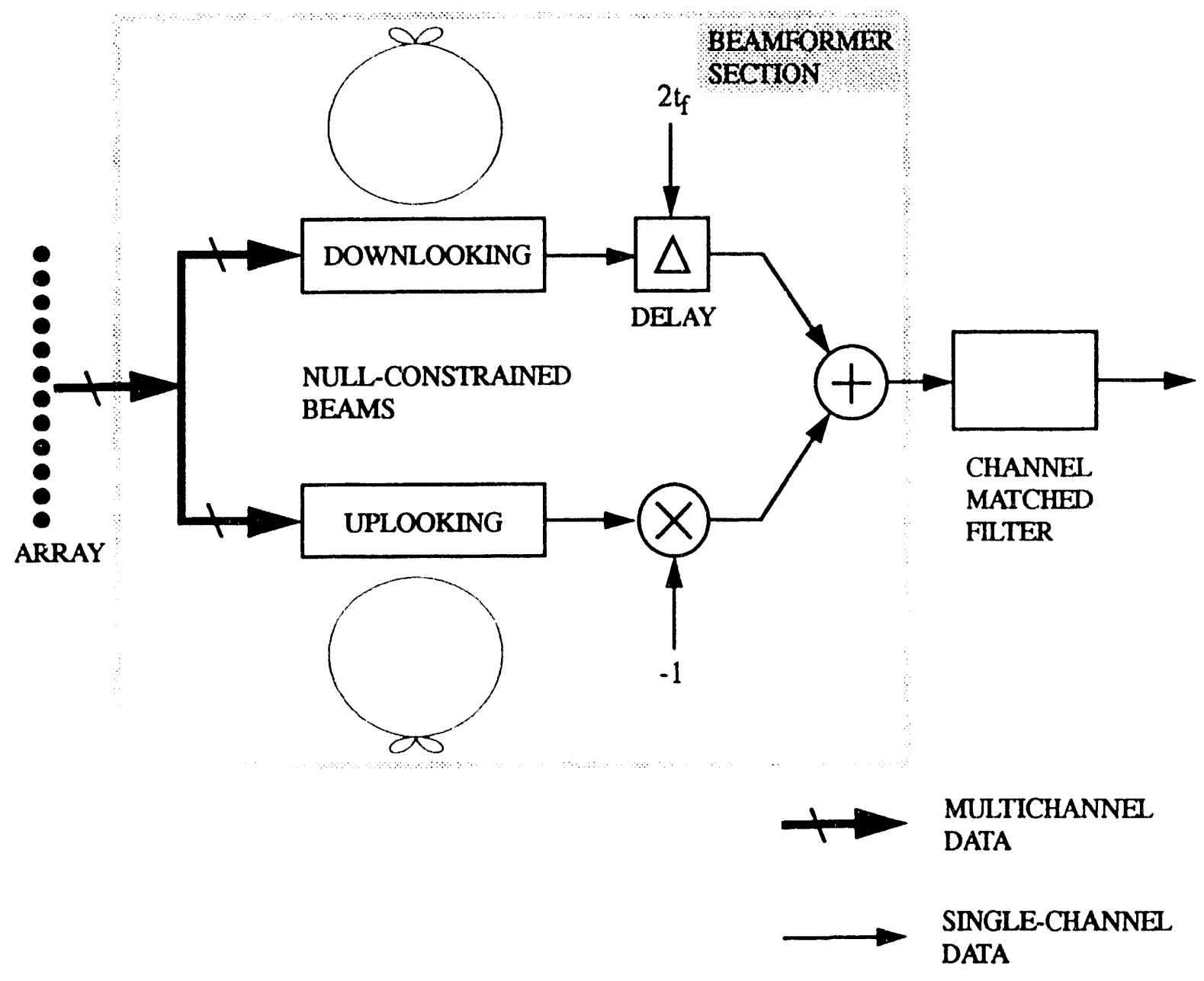

Figure 10 The matched field processor is composed of two independent sections: a "beamformer" section, which accepts the hydrophone waveforms as input and produces a single "beam" waveform, followed by a scalar channel matched filter. The two components can be designed independently; only the channel matched filter requires knowledge of the bottom characteristics; and only the "beamformer" section requires knowledge of the array geometry. 


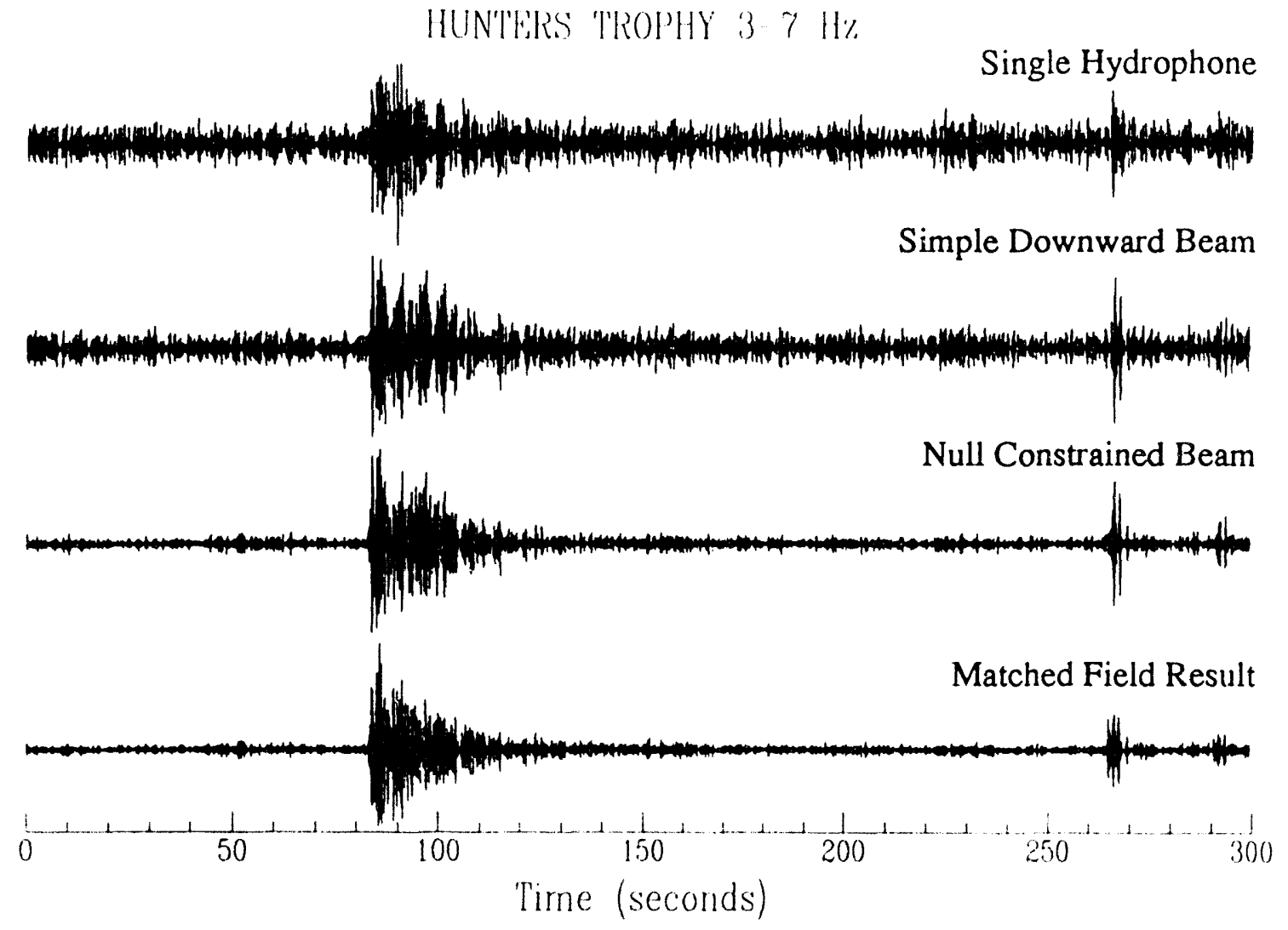

Figure 11 Results of processing HUNTERS TROPHY data in the lower frequency band 3-7 Hz show little improvement with a simple downward-directed beam when compared with a single hydrophone, but significant noise reductions with the null-constrained beam and matched field processing. The simple beam fails to increase SNR significantly because the array aperture ( 165 meters) is only $1 / 2$ wavelength at $5 \mathrm{~Hz}$. The horizontal null helps reduce noise substantially; and further gains are achieved using the matched field approach. Although the ambient acoustic noise is reduced somewhat by matched field processing, the transients between 250 and 300 seconds are reduced significantly. 


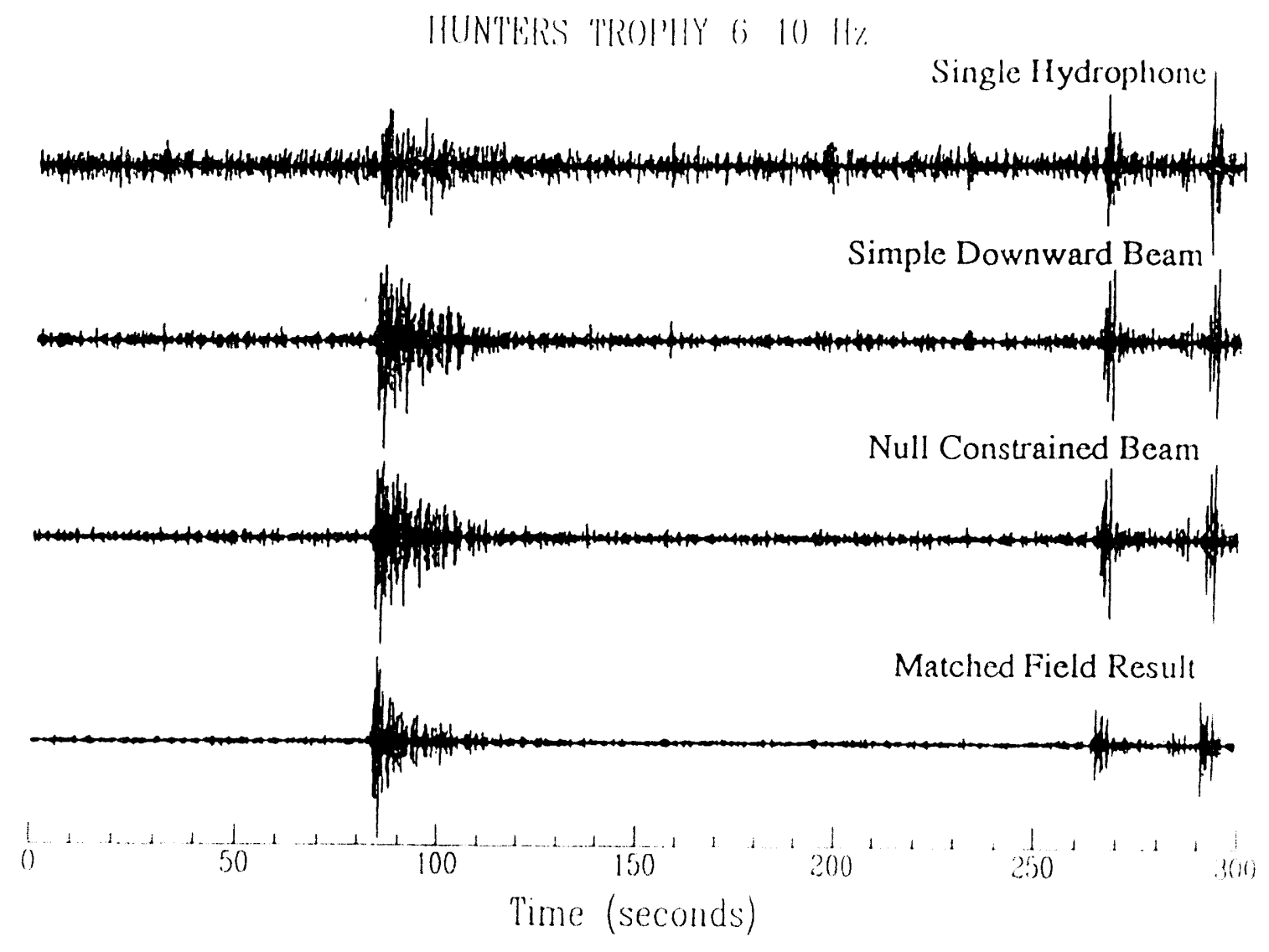

Figure 12 HUNTERS TROPHY processing results in the upper frequency band $6-10 \mathrm{~Hz}$ show greater improvement with a simple downward-directed beam when compared with a single hydrophone, because the array is a full wavelength long in this frequency band. The matched field processing result is significantly better than either of the beams, with greater ambient noise reduction compared to the peak signal and better suppression of the latetime transients. 


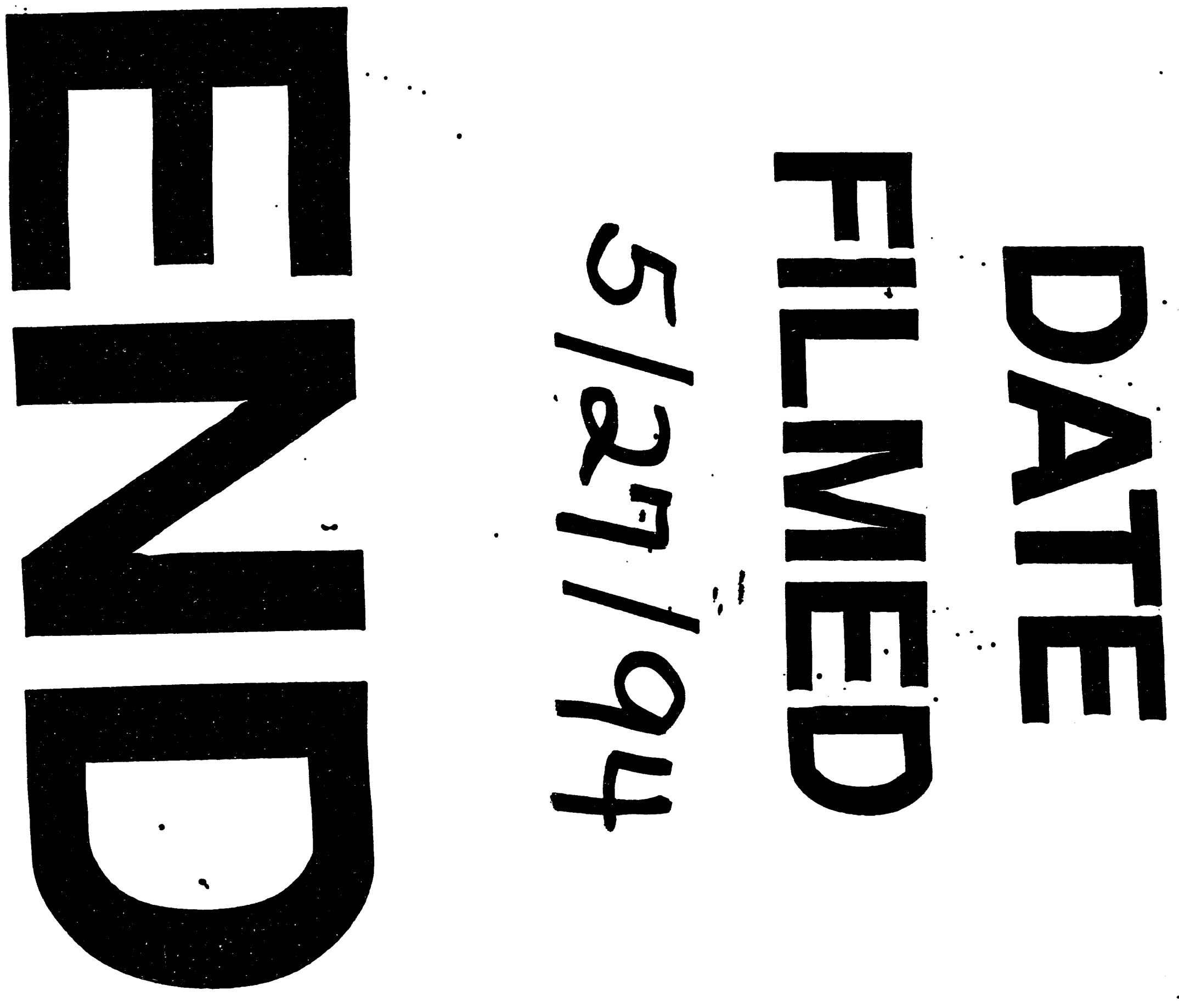


